\title{
Application of One-Dimensional Shallow Water Model to Flows in Open Channels with Bends
}

\author{
Kei Ishida ${ }^{1}$, Koichi Unami ${ }^{2}$ and Toshihiko Kawachi ${ }^{3}$
}

\begin{abstract}
Understanding water flows in open channels is of great importance in agricultural engineering because water is necessary for farming. Although water flow is fundamentally a three-dimensional (3D) phenomenon, onedimensional (1D) models are frequently used in numerical simulations of open channel flows because more detailed data and a greater computational cost are required for higher-dimensional models. Open channels include various structures. A bend is one such structure; however, the effects of a bend are usually neglected in 1D models. Therefore, a mathematical model of the effect of a bend on open channel flows is derived, and a numerical model of 1D flows in open channels with bends is shown by combining the mathematical model with the earlier model. The numerical model is applied to flows in two sets of experiments. The computed results are compared with the experimental data. The first set of experiments for steady-state flows in an open channel with two bends is conducted in this study; the second set of experiments was carried out as part of the Concerned Action of Dam Break Modelling project by Frazão et al. (1999). Dam break flows in bend channels were thus investigated. The comparison between the computed results and the experimental data indicates that the $1 \mathrm{D}$ numerical model can suitably reconstruct flows in open channels with bends.
\end{abstract}

Keywords: Open channel; Bend; One-dimensional model; Shallow water equations; Finite element and volume method

\section{Introduction}

Understanding water flows in open channels such as rivers, irrigation canals, and furrows is of great importance in agricultural engineering, especially in countries where irrigated agriculture is carried out, because water is necessary for farming. Recently, numerical simulations have been widely used to understand open channel flows. In one such approach, a mathematical model describing water flow by partial differential equations is solved numerically. Although water flow is fundamentally a three-dimensional (3D) phenomenon, one-dimensional (1D) mathematical models derived using some approximations are frequently used for numerical simulations of open channel flows because more detailed data and a greater computational cost are required for higher-dimensional models. Although 1D mathematical models are useful, they cannot represent some of the phenomena that are observed in open channels. Open channels include various structures such as weirs, sills, gates, drops, junctions, and bends. When 1D models are used, all structures except bends are usually treated as boundary conditions, and the effects of bends are neglected.

There are few works focusing on water flows in open channels with bends. Frazão et al. (1999) studied dam break flows in experimental channels with a $90^{\circ}$ and $45^{\circ}$ bend as part of the Concerned Action on Dam Break Modelling (CADAM) project in order to validate numerical models. They observed

\footnotetext{
${ }^{1}$ Ph.D. Student, Graduate School of Agriculture, Kyoto University, Kitashirakawa-oiwake-cho, Sakyo-ku, Kyoto 606-8502, Japan (Corresponding Author) E-mail: kei_i@kais.kyoto-u.ac.jp

${ }^{2}$ Associate Professor, Graduate School of Agriculture, Kyoto University, Kitashirakawa-oiwake-cho, Sakyo-ku, Kyoto 606-8502, Japan

${ }^{3}$ Professor, Graduate School of Agriculture, Kyoto University, Kitashirakawa-oiwake-cho, Sakyo-ku, Kyoto 606-8502, Japan
}

the water level at some points using gauges after dam break flows occurred on a dry and a wet bed. Then, they compared these results with those computed by the Boltzmann model. Experimental data have been utilized to validate 2D or 3D numerical models in most studies focusing on water flows in open channels with bends. Various 2D shallow water models have been developed and applied to dam break flows in bend channels. Zhou et al. (2004) developed a high-resolution Gudnov-type cut cell method with transmissive boundary conditions. Kim et al. (2010) modified the cut cell method and applied it to dam break flows. Liang et al. (2004) proposed an adaptive quadtree grids approach and validated it for dam break flows in a $90^{\circ}$ bend channel. Zhang et al. (2011) developed a space-time conservation element and solution element (CE/SE) method for 2D dam break problems. These $2 \mathrm{D}$ approaches were in good agreement with the experimental data. Ferrari et al. (2009) applied a 3D smooth particle hydrodynamics method to dam break flows in a bend channel; however, this model yielded less accurate results compared with a 2D model. Frazão and Zech (2002) used high-speed cameras to image dam break flows in a $90^{\circ}$ bend channel with a dry bed. The flow velocity of the surface was acquired by the Voronoi particle tracking velocimetry technique. The side walls of the channel were made of glass, and this enabled the change in water level to be recorded. Moreover, they utilized a 1D and 2D hybrid model to simulate dam break flows in the channel and compared the results computed by the hybrid model with the experimental data obtained using high-speed cameras and with those computed by a 2D model. In the hybrid model, a 1D approach was used for the straight section of the channel, whereas a $2 \mathrm{D}$ model was used for the bend. The hybrid model was less accurate than the $2 \mathrm{D}$ model. In partic- 
ular, the celerity of the wave that was reflected on the bend was estimated to be much higher by the hybrid model than by the $2 \mathrm{D}$ model and the experimental data. However, the hybrid model has a smaller computational cost than the $2 \mathrm{D}$ model. Biscarini et al. (2010) solved the Reynolds-averaged Navier-Stokes (RANS) equations three dimensionally by the volume of fluid (VOF) method and applied the 3D model to dam break flow in a $90^{\circ}$ bend channel with a dry bed. The model agreed well with the experimental data recorded using high-speed cameras compared with a $2 \mathrm{D}$ shallow water model. This indicates that the flow in a bend is 3D rather than 2D. Thus, 2D and 3D models having sufficient accuracy have been developed to simulate water flows in a bend channel. On the other hand, the 1D and 2D hybrid model is one of the good approaches for simulating water flows in a bend channel with less computational cost, despite some disadvantages. Sanders et al. (2001) noted that it is difficult to apply the hybrid model to channels whose cross sections are not rectangular, trapezoidal, or triangular, but arbitrary or natural.

This study aims to apply a 1D numerical model to simulations of water flows in open channels with bends. The finite element and volume method (FEVM) is used for the simulations because it can be applied to a wide range of simulations of 1D open channel flows. The FEVM was developed by Unami et al. (1996) as a 1D network flow model. Then, Ishida et al. (2011) proposed a stabilized scheme for the FEVM and applied it to the simulation of flows in a farmland where the channels have junctions and sudden changes in width and slope. A mathematical model of the effect of a bend on open channel flows is derived, and a 1D numerical model including the effects of bends is developed by combining the mathematical model for bends with the earlier model developed by Ishida et al. (2011). In this study, steady-state flows in an open channel with bends are investigated. The experimental conditions are set such that transient flows occur. The positions of hydraulic jumps are measured, and the experimental results are compared with those computed by the proposed model in order to validate the model in steadystate flow conditions. Next, dam break flows in open channels with a $90^{\circ}$ or $45^{\circ}$ bend are solved using the model, and the results are compared with experimental data in order to validate the model in unsteady-state flow conditions. Moreover, dam break flows are computed with the earlier model neglecting the effects of bends. A comparison of the results computed by the proposed and earlier models show the advantage of the former, which includes the effects of bends.

\section{Open channel flow model}

\subsection{Mathematical Model}

The flow of water in an open channel is three dimensional. However, when the flow along the open channel bed is dominant, it can be modeled one dimensionally, and the direction of the flow is called the main flow direction. In 1D models, the horizontal coordinate of the open channel bed is projected onto the local curvilinear abscissa $x$. If $x$ is, or is estimated to be, smooth in a $3 \mathrm{D}$ Euclidean space $\mathbb{R}^{3}$, the main flow direction continuously changes in the open channel. The flow in the open channel is described by the well-known 1D shallow water equations (SWEs) in conservative forms assuming that water is incompressible and that the pressure distribution is hydrostatic. The 1D SWEs consist of the continuity equation and the momentum equation representing the conservation laws of mass and momentum, respectively. The continuity equation is written as

$$
\frac{\partial A}{\partial t}+\frac{\partial Q}{\partial x}=q
$$

where $t$ is the time, $Q$ the discharge, $q$ the lateral inflow discharge per unit length of the channel, and the wetted crosssectional area $A$ is a monotone increasing function of the water level $\eta$. The momentum equation is expressed as

$$
\frac{\partial Q}{\partial t}+\frac{\partial F}{\partial x}=S
$$

where the flux $F$ and the source term $S$ are respectively defined as

$$
F=\frac{\beta Q^{2}}{A}
$$

and

$$
S=-g A \frac{\partial \eta}{\partial x}-g A S_{f}
$$

where $\beta$ is the Coriolis coefficient, $g$ the acceleration due to gravity, and $S_{f}$ is the friction slope, which can be estimated using the Manning's equation

$$
S_{f}=\frac{n^{2} Q|Q| P^{\frac{4}{3}}}{A^{\frac{10}{3}}}
$$

where $n$ is the Manning's roughness coefficient. When the roughness is not uniform in a cross section of the channel, Manning's roughness coefficient for the cross section should be calculated by

$$
n=\frac{\left|\sum_{m=1}^{N^{D}}\left(P_{m} n_{m}^{1.5}\right)\right|^{\frac{2}{3}}}{P^{\frac{2}{3}}}
$$

where $N^{D}$ is the number of divisions whose the Manning's roughness coefficients differ from each other in the cross section, and $P_{m}$ and $n_{m}$ are the wetted perimeter and the Manning's roughness coefficient of the $m$ th division, respectively.

If the open channel includes bends, $x$ is continuous but not smooth. The direction of the cross-sectional average flow velocity along the main flow direction suddenly changes at the bends whereas the water level continuously changes. In other words, the point of any bend is a singular point in $x$. In order to model the flow in bend channels, the physical quantity through a bend must be estimated. Figure 1 shows a test domain including a bend. The test domain is divided by the bend into two subdomains, denoted by $\mathcal{V}_{1}$ and $\mathcal{V}_{2}$. The wetted part of the cross section of the bend is denoted by $\mathcal{D}$. The conservation law of mass is satisfied at the bend, and the discharge through $\mathcal{D}$ is given by

$$
Q^{\mathcal{D}}=\int_{\mathcal{D}} \mathbf{v} \cdot \mathbf{n} \mathrm{d} \mathcal{D}
$$




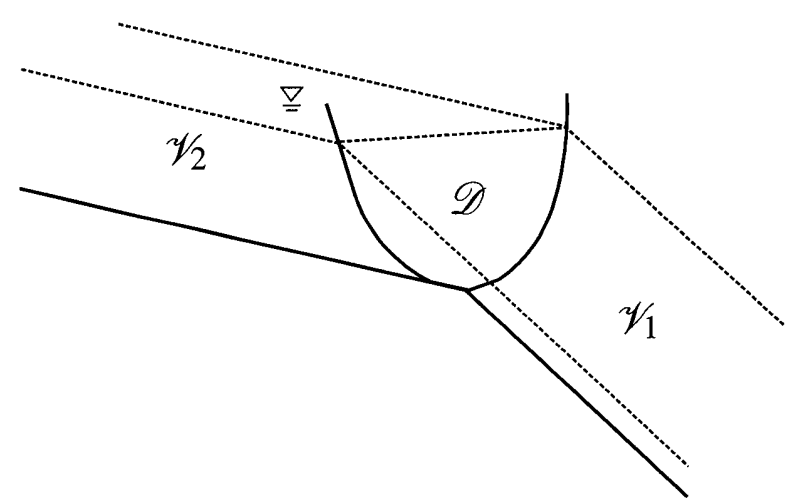

Figure 1: Test domain including a bend

where $\mathbf{v}$ is the absolute velocity vector of water and $\mathbf{n}$ is the normal unit vector of $\mathcal{D}$ whose direction is from $V_{1}$ to $V_{2}$. On the other hand, the flux at a point on $\mathcal{D}$

$$
f^{\mathcal{D}}=(\mathbf{v} \cdot \mathbf{n}) \mathbf{v}
$$

is given. By projecting $f^{\mathcal{D}}$ on $x$, the flux along the main flow direction at a point on $\mathcal{D}$

$$
f^{\mathcal{D}, \mathbf{X}_{1}}=(\mathbf{v} \cdot \mathbf{n}) \mathbf{v} \cdot \mathbf{X}_{1}
$$

is calculated, where $\mathbf{X}_{1}$ is the unit vector along $x$ at $\mathcal{D}$ in $\mathcal{V}_{1}$. Integration of $f^{\mathcal{D}, \mathbf{X}}$ over $\mathcal{D}$ yields the flux at $\mathcal{D}$ in $\mathcal{V}_{1}$

$$
\left.F^{\mathcal{V}_{1}}\right|_{\mathcal{D}}=\int_{\mathcal{D}}(\mathbf{v} \cdot \mathbf{n}) \mathbf{v} \cdot \mathbf{X}_{1} \mathrm{~d} \mathcal{D}=\left.\beta V^{\mathcal{V}_{1}}\right|_{\mathcal{D}} Q^{\mathcal{D}}
$$

where $\left.V^{V_{1}}\right|_{\mathcal{D}}$ is the cross-sectional mean velocity along $\mathbf{X}_{1}$ at $\mathcal{D}$ in $\mathcal{V}_{1}$. Similarly, the flux at $\mathcal{D}$ in $\mathcal{V}_{2}$

$$
\left.F^{\mathcal{V}_{2}}\right|_{\mathcal{D}}=-\int_{\mathcal{D}}(\mathbf{v} \cdot \mathbf{n}) \mathbf{v} \cdot \mathbf{X}_{2} \mathrm{~d} \mathcal{D}=\left.\beta V^{\mathcal{V}_{2}}\right|_{\mathcal{D}} Q^{\mathcal{D}}
$$

is obtained, where $\mathbf{X}_{2}$ is the unit vector along $x$ at $\mathcal{D}$ in $\mathcal{V}_{2}$, and $\left.V^{\mathcal{V}_{2}}\right|_{\mathcal{D}}$ is the cross-sectional mean velocity along $\mathbf{X}_{2}$ at $\mathcal{D}$ in $\mathcal{V}_{2}$.

If water flows from $\mathcal{V}_{1}$ into $\mathcal{V}_{2}$, the relationship between $\left.V^{\mathcal{V}_{1}}\right|_{\mathcal{D}}$ and $\left.V^{\mathcal{V}_{2}}\right|_{\mathcal{D}}$ is represented by

$$
\max \left(\left.\cos \theta_{\left.\mathbf{x}_{1}, \mathbf{x}_{2}, 0\right)} V^{\mathcal{V}_{1}}\right|_{\mathcal{D}}=\left.V^{V_{2}}\right|_{\mathcal{D}}\right.
$$

where $\theta \mathbf{x}_{1}, \mathbf{x}_{2}$ is the angle between $\mathbf{X}_{1}$ and $\mathbf{X}_{2}$. Substitution of Eqs. (10) and (11) into Eq. (12) yields

$$
\left.\max \left(\cos \theta_{\mathbf{x}_{1}, \mathbf{x}_{2}}, 0\right) F^{\mathcal{V}_{1}}\right|_{\mathcal{D}}=\left.F^{\mathcal{V}_{2}}\right|_{\mathcal{D}}
$$

If $\theta \mathbf{x}_{1}, \mathbf{X}_{2}=0$, Eq. (13) is reduced to

$$
\left.F^{\mathcal{V}_{1}}\right|_{\mathcal{D}}=\left.F^{\mathcal{V}_{2}}\right|_{\mathcal{D}}
$$

i.e., Eq. (13) is also satisfied if $x$ belongs to $C^{m}$ with $m=0$, 1 . If water flows from $\mathcal{V}_{2}$ into $\mathcal{V}_{1}$, the relation

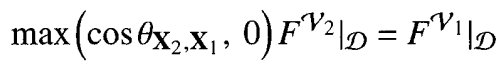

is obtained in the same manner

\subsection{Numerical model}

For it to be applicable to open channels with bends, Eq. (1) is transformed to the weak form

$$
\int_{\Omega} \varphi T \frac{\mathrm{d} \eta}{\mathrm{d} t} \mathrm{~d} x-\int_{\Omega} \frac{\partial \varphi}{\partial x} Q \mathrm{~d} x=\int_{\Omega} \varphi q \mathrm{~d} x-[\varphi Q]_{\Gamma}
$$

where $T=\frac{\partial A}{\partial \eta}$ is the width of the water surface, $\Omega$ the domain constituting the entire channel, $\Gamma$ the boundary of $\Omega$, and $\varphi$ is any weight belonging to $H^{1}(\Omega)$.

In order to numerically solve the SWEs with the FEVM, the domain $\Omega$ is divided into $N_{E}$ elements by $N_{N}$ nodes so that any singular point falls on one of the nodes. The nodes in $\Omega$ are numbered along the direction of $x$, and the element bound by the $i$ th and $(i+1)$ th nodes is numbered $k$. To discretize the unknowns, values of $\eta$ are assigned at the nodes whereas those of $Q, \beta$, and $n$ are assigned in the elements. The subscripts of $Q$ represent the elemental values. The function $T$ is linearly interpolated in each element, and $T_{k, i}$ denotes $T$ at the end of the $k$ th element falling on the $i$ th node. Then, substituting the standard bases of element-wise linear functions into $\varphi$ of Eq. (16) results in the nodal equations

$$
\begin{cases}I(k, i)-Q_{k}=Q_{i}^{*} & (i=1) \\ I(k-1, i)+I(k, i)+Q_{k-1}-Q_{k}=Q_{i}^{*} & \left(i=2 \sim N_{N}\right) \\ I(k-1, i)+Q_{k-1}=Q_{i}^{*} & \left(i=N_{N}\right)\end{cases}
$$

where $Q_{i}^{*}$ is the inflow discharge concentrated at the $i$ th node, and $I(k, i)$ is the integral given by

$$
\begin{aligned}
I(k, i)=\Delta x_{k}\left(\frac{T_{k, \iota(k, i)}}{12}+\right. & \left.\frac{T_{k, i}}{12}\right) \frac{\mathrm{d} \eta_{\iota(k, i)}}{\mathrm{d} t} \\
& +\Delta x_{k}\left(\frac{T_{k, \iota(k, i)}}{12}+\frac{T_{k, i}}{4}\right) \frac{\mathrm{d} \eta_{i}}{\mathrm{~d} t}
\end{aligned}
$$

where $\Delta x_{k}$ is the length of the $k$ th element, and $\iota(k, i)$ is the number of the node binding the $k$ th element with the $i$ th node. A system of linear equations consisting of Eq. (17) with Eq. (18) for each node is solved to obtain ordinary differential equations

$$
\frac{\mathrm{d} \eta_{i}}{\mathrm{~d} t}=f_{i}
$$

where $f_{i}$ is a known function at every temporal stage.

The finite volume method is applied to the momentum equation. Integration of Eq. (2) over a generic $k$ th element $\Omega_{k}$ yields

$$
\frac{\mathrm{d}}{\mathrm{d} t} \int_{\Omega_{k}} Q \mathrm{~d} \Omega_{k}+[F]_{\Gamma_{k}}=\int_{\Omega_{k}} S \mathrm{~d} \Omega_{k}
$$

where $\Gamma_{k}$ is the boundary of $\Omega_{k}$. The wetted cross-sectional areas estimated at the upstream and downstream ends of $\Omega_{k}$ in terms of the flow direction are denoted by $A_{k}^{\text {up }}$ and $A_{k}^{\text {down }}$, respectively. An elemental Froude number $F r_{k}$ is defined by

$$
F r_{k}^{2}=\frac{\beta_{k} T_{k}^{\text {down }} Q_{k}^{2}}{g\left(A_{k}^{\text {down }}\right)^{3}}
$$




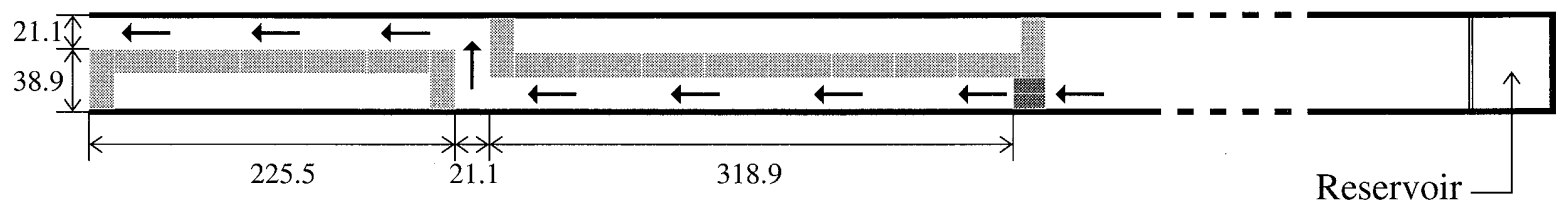

: Concrete block $\quad$ : Brick $\leftarrow$ : Flow direction

Figure 2: Plan view of the channel with two $90^{\circ}$ bends for steady-state simulation

where $T_{k}^{\text {down }}$ is $T$ at the downstream end of $\Omega_{k}$, to evaluate an elemental velocity $V_{k}$ as

$$
V_{k}=\frac{Q_{k}}{(1-\omega) A_{k}^{\text {down }}+\omega A_{k}^{\text {up }}}
$$

where $\omega$ is the weight calculated as

$$
\omega=\max \left(1-\frac{1}{F r_{k}^{2}}, 0\right) .
$$

Using this $V_{k}$ with the elemental values of $\beta$ and $Q$, an elemental flux $F_{k}^{E}$ is defined as

$$
F_{k}^{E}=\beta_{k} Q_{k} V_{k}
$$

The flux at a boundary of a element is estimated from the elemental fluxes of the two elements connected to the boundary. Both the $(k-1)$ th and the $(k+1)$ th elements, which are connected to the $k$ th element, are together denoted by the $\kappa$ th element. If water flows from the $\kappa$ th element into the $k$ th element, the flux at the downstream node of the $\kappa$ th element is given by the elemental flux of the $\kappa$ th element, and the flux at the upstream node of the $k$ th element is derived from Eqs. (13) and (15) as

$$
F_{k}^{\mathrm{up}}=\max \left(\cos \theta_{k, \kappa}, 0\right) F_{\kappa}^{E}
$$

where $\theta_{k, K}$ is the angle between the main flow directions of the $k$ th element and the $\kappa$ th element. Discretizing by a 1D model causes a case in which flows in two adjacent elements, the $k$ th and the $\kappa$ th elements, are in opposing directions, i.e., toward each other. In this case, the fluxes at the boundary of the $k$ th and the $\kappa$ th elements also affect each other. By considering Eqs. (13) and (15), the flux at the downstream node of the $k$ th element is estimated as

$$
F_{k}^{\text {down }}=F_{k}^{E}-\min \left(\cos \theta_{k, \kappa}, 0\right) F_{\kappa}^{E} .
$$

Finally, Eq. (20) is arranged as

$$
\frac{\mathrm{d} Q_{k}}{\mathrm{~d} t}=-\frac{1}{\Delta x(k)}[F]_{\Gamma_{k}}+S_{k}
$$

by calculating the integration where $[F]_{\Gamma_{k}}$ is evaluated using $F_{k}^{\text {up }}$ and $F_{k}^{\text {down }}$.

\section{Applications}

For validation, the proposed model is applied to steady-state flows in a open channel with two $90^{\circ}$ bends and unsteadystate flows in open channels with a bend. The computed results are compared with experimental ones. The former experiment is conducted to validate the numerical model in steady-state flow conditions. The latter experiment involving dam break flows in bend channels was carried out at the Université Catholique de Louvain by the CADAM group (Frazão et al., 1999). Moreover, the earlier model that neglected the effects of bends was also applied to dam break flows. A comparison of the results computed by the proposed and earlier models is conducted to show the advantages of the former.

\subsection{Steady-state flow}

There is a flume in Kyoto University, having a length and width of $20 \mathrm{~m}$ and $0.60 \mathrm{~m}$, respectively. The wall and the bed of this flume are made of glass and steel, respectively. The flume is designed such that its Manning's coefficient is 0.01 $\mathrm{m}^{-\frac{1}{3}} \mathrm{~s}$. There is a reservoir at the upstream end of the flume and a partition between the reservoir and the flume. Water is supplied to the reservoir using a pump, and the water flows over the partition and into the flume. At the downstream end, there is a weir whose height can be adjusted. The slope of the channel can also be adjusted from 0 to $1 / 100$.

For the experimental tests, concrete blocks are arranged on the flume to create a control section regulating the flow (Figure 2). The control section forms a test channel with two $90^{\circ}$ bends. The two bends are numbered from the upstream side. The width of the test channel is $0.21 \mathrm{~m}$. The distance from the downstream end to bend 2 is $2.26 \mathrm{~m}$. Two bricks are placed at the upstream end of the test channel, which is 3.19 $\mathrm{m}$ away from bend 1 , such that water flows over the bricks and the effect of flow contraction due to a sudden change in the channel width is limited. The slope is set to $1 / 100$. The Manning's coefficient of the concrete blocks is estimated to be $0.012 \mathrm{~m}^{-\frac{1}{3}} \mathrm{~s}$ from the water depth at the point where the flow is uniform, and therefore, the Manning's coefficient of the test channel is calculated by Eq. (6). The height of the weir at the downstream end was set to $0 \mathrm{~m}$, and water dropped freely. Water discharge was set to be $3.70,4.55$, and $5.25 \mathrm{~L} / \mathrm{s}$ by regulating the pump.

In each of the cases, water was discharged continuously until the water flow attained a steady state. Then, water flow was supercritical downstream of the bricks and subcritical in the bends, and it transited conversely from a subcritical state to a supercritical state downstream of bend 2. Hence, a hydraulic jump arose between bend 1 and the bricks. The hydraulic jump is estimated to be a weak jump or an oscillating jump because rollers occurred at the front of the hydraulic jump and the water surface oscillated up to bend 1 . The position of the hydraulic jump was measured, but it was unstable. 


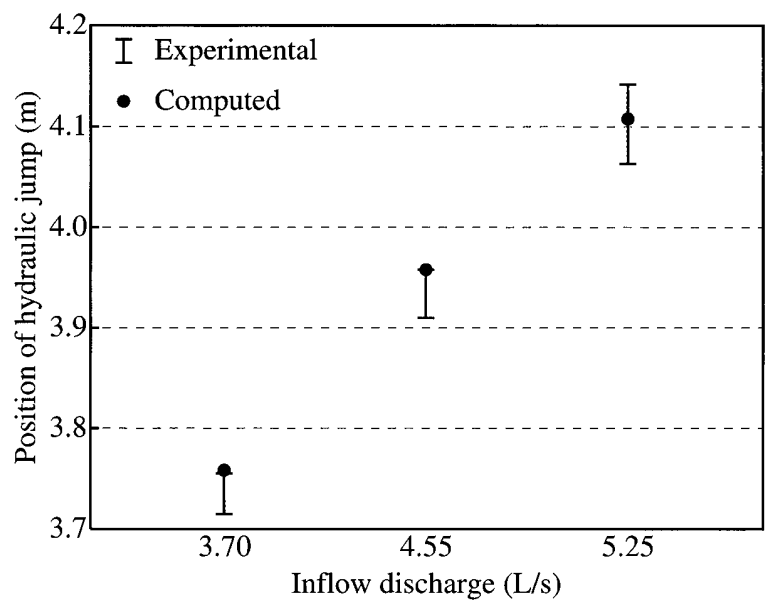

Figure 3: Position of the front of the hydraulic jump in each inflow discharge

Waves of the hydraulic jump were reflected on the concrete block of the bend. The waves caused oscillations and fluctuated the front of the hydraulic jump. Another reason for the instability is oscillation of the water discharge supplied by the pump. The position of the hydraulic jump, that is, the position where the water depth is highest immediately downstream of the beginning of the hydraulic jump, is shown in Figure 3. This position is the distance from the downstream end.

A computational domain is set to be the test channel from the bricks to the downstream end of the experimental channel for numerical simulations, and the proposed numerical model is applied to the computational domain. An open boundary is imposed at the downstream end, and constant discharges, $3.70,4.55$, and $5.25 \mathrm{~L} / \mathrm{s}$, are supplied at the upstream end. The mesh size is $0.05 \mathrm{~m}$. The time step is 0.01 s. The computed position of the hydraulic jump in each case is shown in Figure 3. The computed positions in the cases of inflow discharges of 4.55 and $5.25 \mathrm{~L} / \mathrm{s}$ are included in the range of positions given by the experiments; that in the case of an inflow discharge of $3.70 \mathrm{~L} / \mathrm{s}$ is slightly larger than the experimental one. Thus, the results computed by the numerical model are consistent enough with the experimental ones, although the model based on SWEs cannot reconstruct oscillations of the hydraulic jumps.

\subsection{Dam break flow}

The dam break experiments were carried out using two open channels, as shown in Figure 4. Each open channel consists of a upstream reservoir and a downstream channel with a bend. In both channels, the wall and the bed are made of glass and steel, respectively. The Manning's roughness coefficients of the wall and the bed are $0.0095 \mathrm{~m}^{-\frac{1}{3}} \mathrm{~s}$ and 0.0195 $\mathrm{m}^{-\frac{1}{3}} \mathrm{~s}$, respectively. The reservoir is a rectangular with dimensions of $2.44 \mathrm{~m} \times 2.39 \mathrm{~m}$, and the bottom level is 0.33 $\mathrm{m}$ lower than the downstream channel (see Figure 4c). The downstream channel has a rectangular cross section of 0.50 $\mathrm{m}$ width. Water can drop at the downstream end. The water level was recorded using gauges. A gate separated the reservoir and the downstream channel before the beginning of each experiment. The gate was suddenly opened to create dam break flows.

\subsubsection{Channel with a $90^{\circ}$ bend}

The shape of the $90^{\circ}$ bend channel is shown in Figure $4 \mathrm{a}$. The downstream channel consists of a 3.92-m-long upstream reach, 2.92-m-long downstream reach, and a $90^{\circ}$ bend. The initial water level in the reservoir was $0.20 \mathrm{~m}$ above the bed level of the downstream channel, and the downstream channel was initially dry. The water level was recorded using six gauges whose locations are shown in Figure 4a. Gauging point $\mathrm{G} 1$ is located in the upstream reservoir; $\mathrm{G} 2, \mathrm{G} 3$, and $\mathrm{G} 4$ are located in the upstream reach from the bend; and G5 and G6 are located in the downstream reach. The experimental data are plotted in Figure 5.

The proposed numerical model is applied to the dam break flow in the $90^{\circ}$ bend channel. An open boundary is imposed at the downstream end. The initial condition in the downstream channel is set to $0.001 \mathrm{~m}$ as a dry condition. The mesh length is $0.05 \mathrm{~m}$. The time step is $0.001 \mathrm{~s}$. The computed results are shown in Figure 5.

The computed celerity in the upstream reach agrees with the experimental data well before the wave reflects on the bend. However, the computed celerity in the downstream reach is faster than the experimental data, and that of the wave reflected on the bend differs slightly from the experimental one. The computed water level is higher than the experimental one at $\mathrm{G} 2$ because the contraction caused by the sudden change in the channel actually influenced the flow at G2 near the upstream reservoir. Moreover, the computed and experimental water levels are different at G5 because the flow after the $90^{\circ}$ bend is $2 \mathrm{D}$ in reality. Overall, the computed water level is in good agreement with the experimental one.

For comparison, the earlier model, neglecting the effects of bends, is also applied with the same conditions. The computed water levels are shown in Figure 5. In the upstream reach from the bend, the wave reflected on the bend, and the backwater effect by the bend cannot be reconstructed by the earlier model although the differences between the computed results by the proposed and earlier models are not large in the downstream reach. The differences reduce with the distance from the bend in the entire channel.

\subsubsection{Channel with a $45^{\circ}$ bend}

The shape of the channel is shown in Figure $4 \mathrm{~b}$. The downstream channel consists of a 4.25-m-long upstream reach, 3.94-m-long downstream reach, and a $45^{\circ}$ bend. The initial water level in the reservoir was $0.25 \mathrm{~m}$ above the bed level of the downstream channel, and the downstream channel was initially dry. The water level was recorded using nine gauges whose locations are shown in Figure $4 b$. Gauging point G1 is located in the upstream reservoir; G2, G3, and G4 are located in the upstream reach from the bend; G5, G6, G7, G8, and G9 are located in the downstream reach. Instead of G5 and G7, G6 is used to for 1D comparisons between the com- 

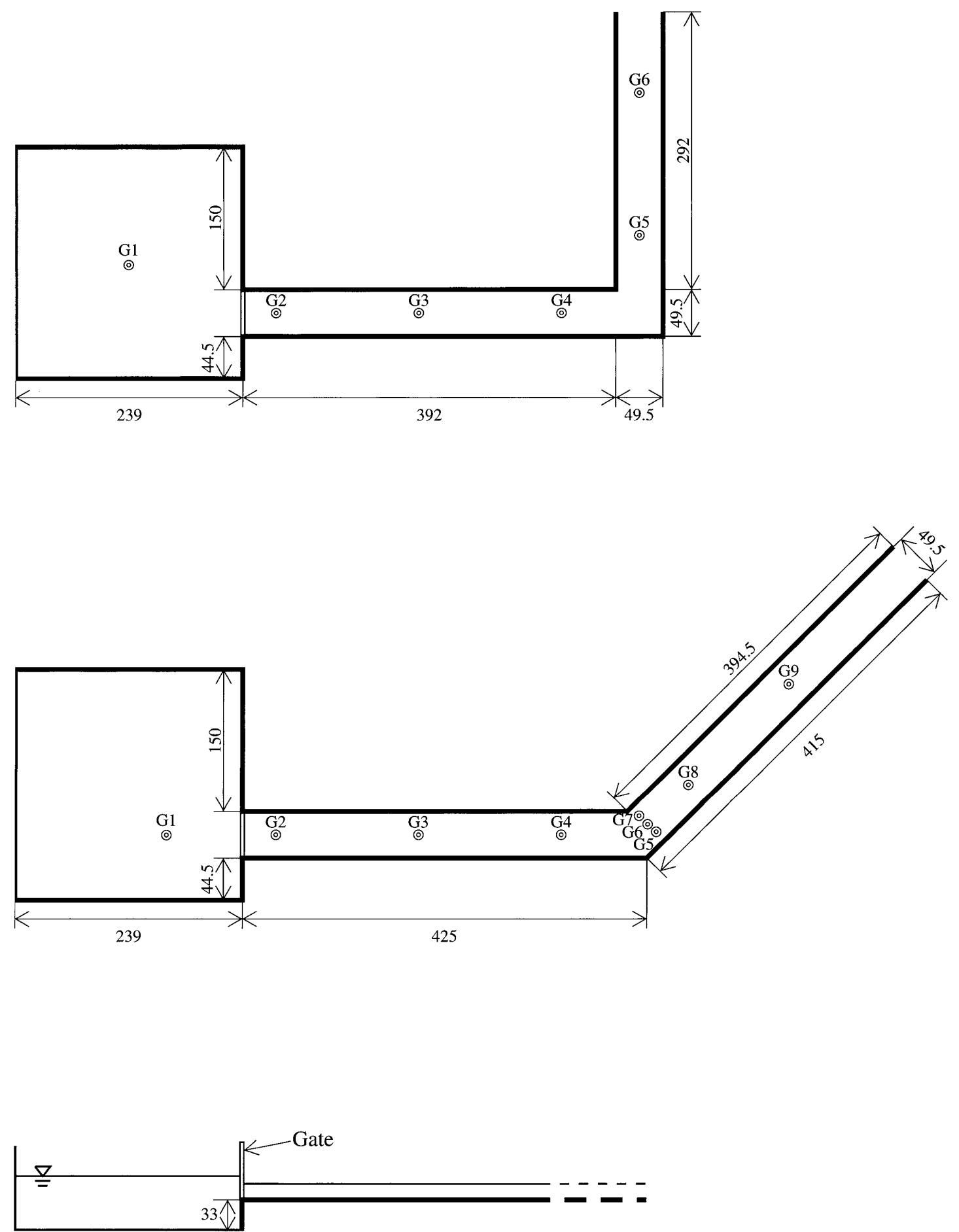

Figure 4: Plan view of channels with a) a $90^{\circ}$ bend and b) a $45^{\circ}$ bend, and c) side view of the upstream reservoir

puted and experimental water levels. The experimental water levels except for G5 and G7 are plotted in Figure 6.

The proposed numerical model is also applied to the dam break flow in the $45^{\circ}$ bend channel. The open boundary is imposed at the downstream end. The initial condition in the downstream channel is set to $0.001 \mathrm{~m}$ as a dry condition. The mesh length is $0.05 \mathrm{~m}$. The time step is $0.001 \mathrm{~s}$. The computed results are shown in Figure 6.
The computed celerity in the upstream reach agrees with the experimental data well before the wave reflects on the bend, but that in the downstream reach is faster than the experimental data, as in the case of the $90^{\circ}$ bend channel. The computed celerity of the wave reflected on the bend is much higher than the experimental one, unlike in the experiments with the $90^{\circ}$ bend channel, because the angle of the bend is $45^{\circ}$, which is smaller than that in the above experiments, 


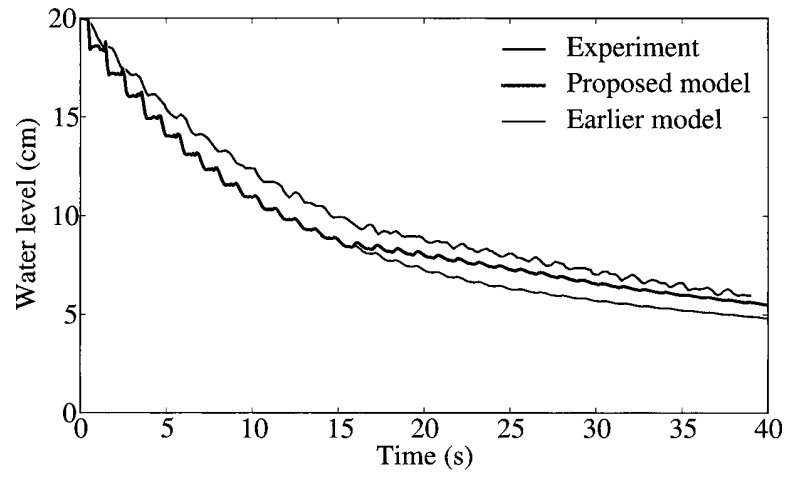

a) G1

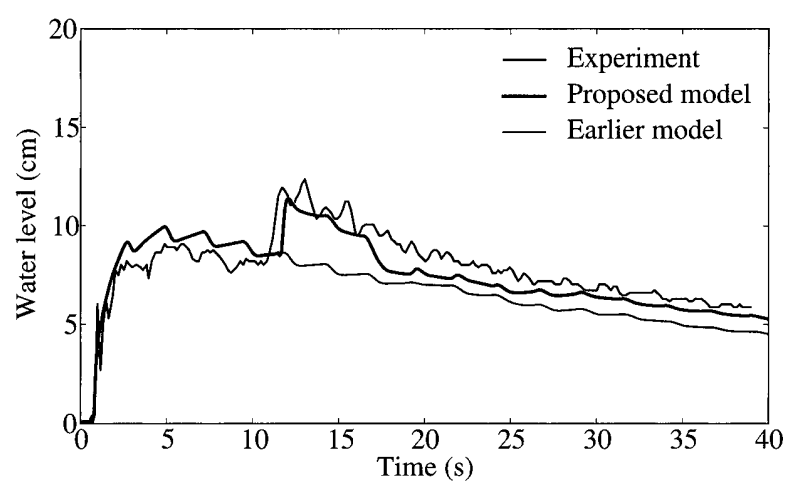

c) G3

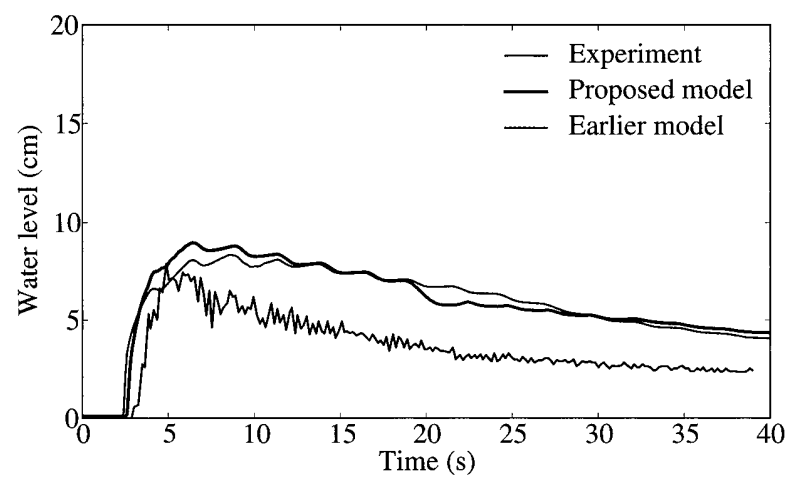

e) G5

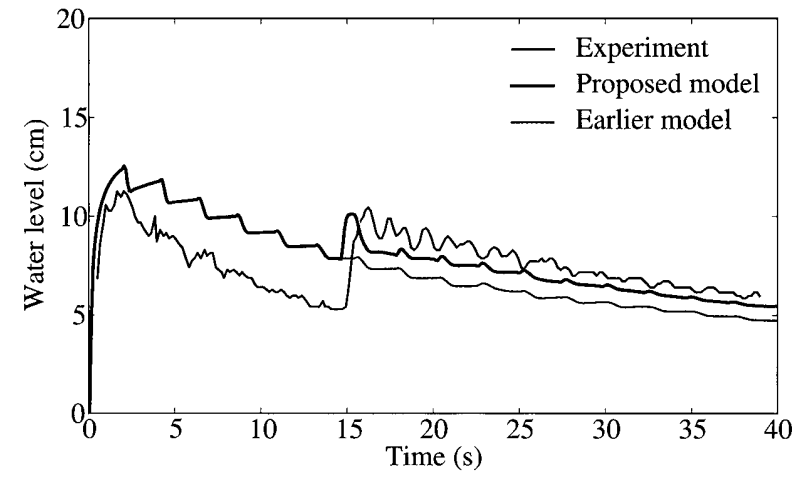

b) G2

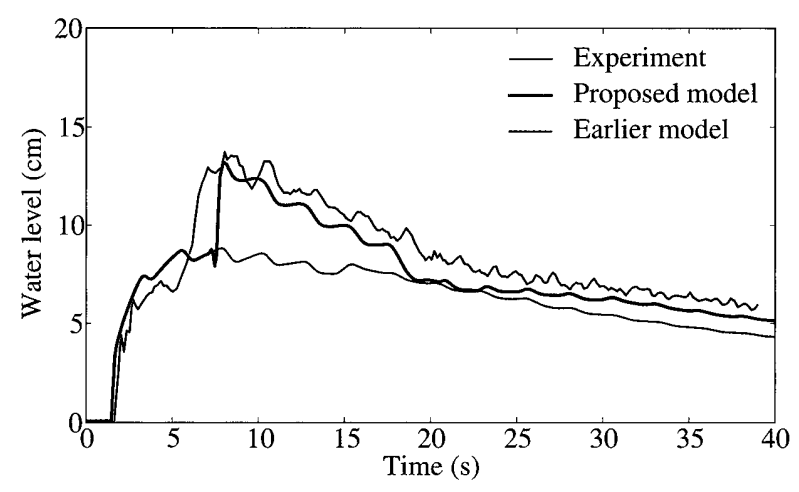

d) $\mathrm{G} 4$

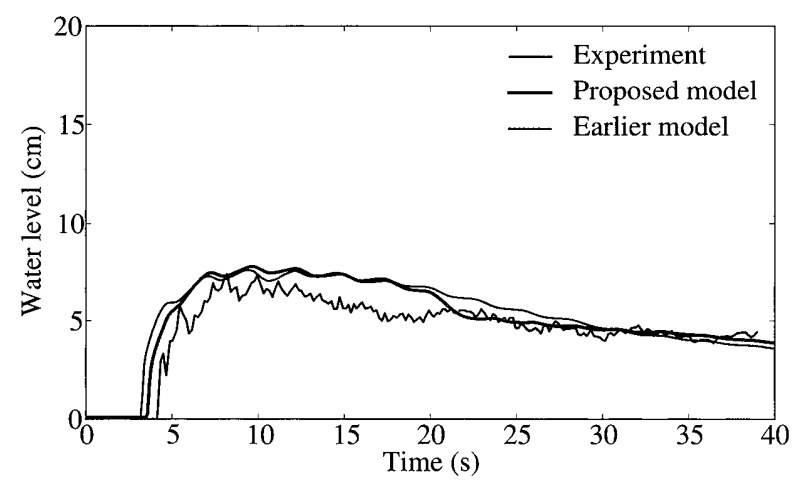

f) $\mathrm{G} 6$

Figure 5: Computed water levels by proposed and earlier models and experimental water level (Frazão et al., 1999) at the six gauging points in the $90^{\circ}$ bend channel

and water in these experiments flows more one dimensionally than in the above experiments.

The earlier numerical model is also applied with the same conditions, whose results are shown in Figure 6. As is the case with the $90^{\circ}$ bend channel, the differences between the computed results by the proposed and earlier models reduce with distance from the bend in the entire channel. The wave reflected on the bend and the backwater effect by the bend cannot be reconstructed by the earlier model in the upstream reach from the bend, whereas the proposed model can reconstruct them. At G6, the water levels by the proposed model better fit the experimental data than those by the ear- lier model, because G6 is located near the bend.

\section{Conclusions}

The mathematical model of water flows at a bend in an open channel has been derived, and this has been combined with a numerical model using the FEVM to construct a numerical model for flows in open channels with bends. The proposed numerical model has been applied to flows in the bend channels, and it has been compared with two sets of experimental data for validation. One of the sets was obtained in this study. The steady-state flows in the two bend channels were investigated in the experiments. The position of the front of 


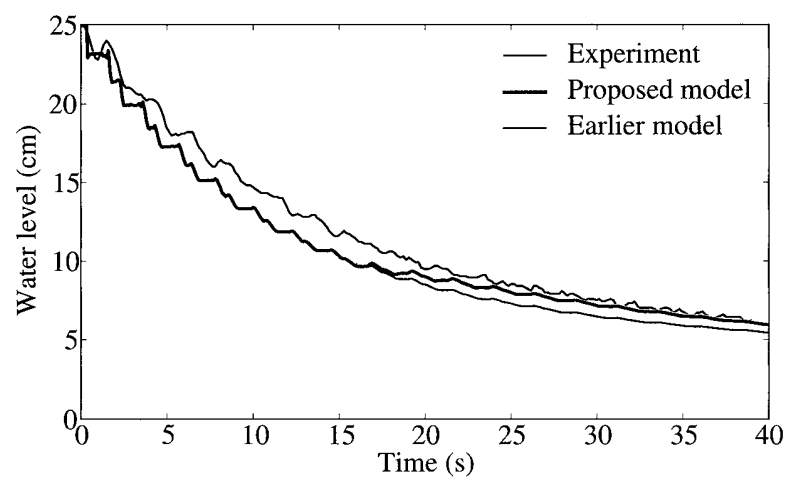

a) G1

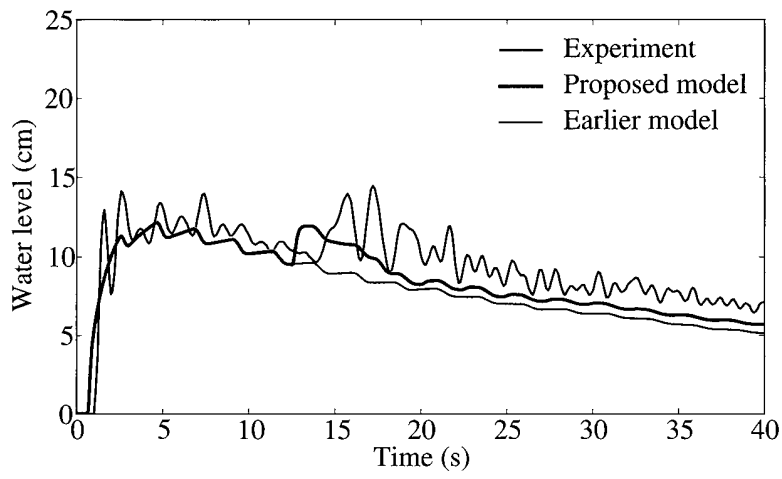

c) G3

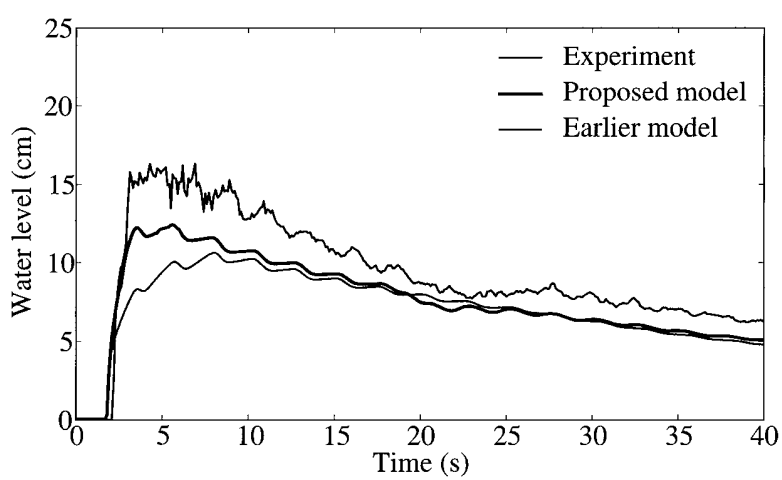

e) G6

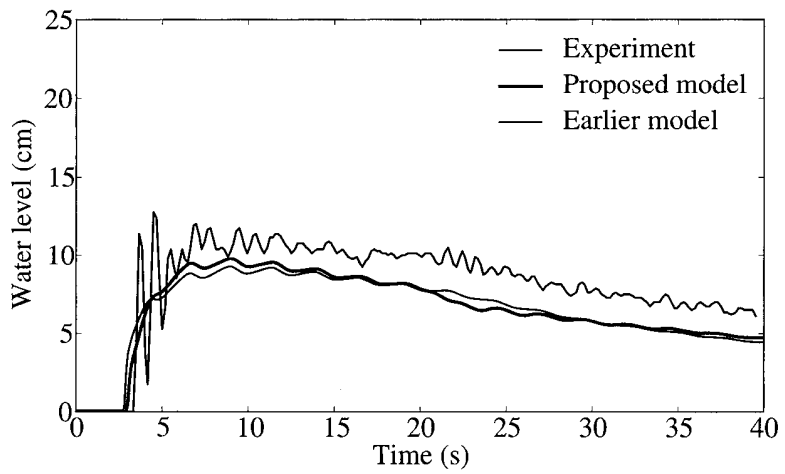

g) G9

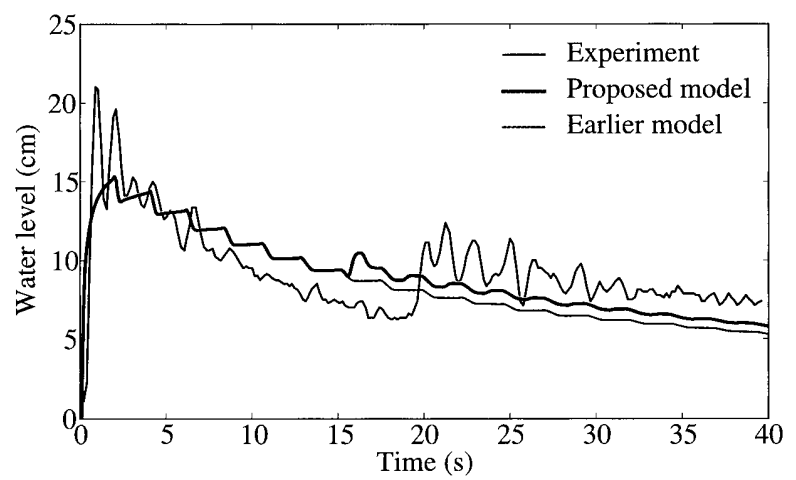

b) G2

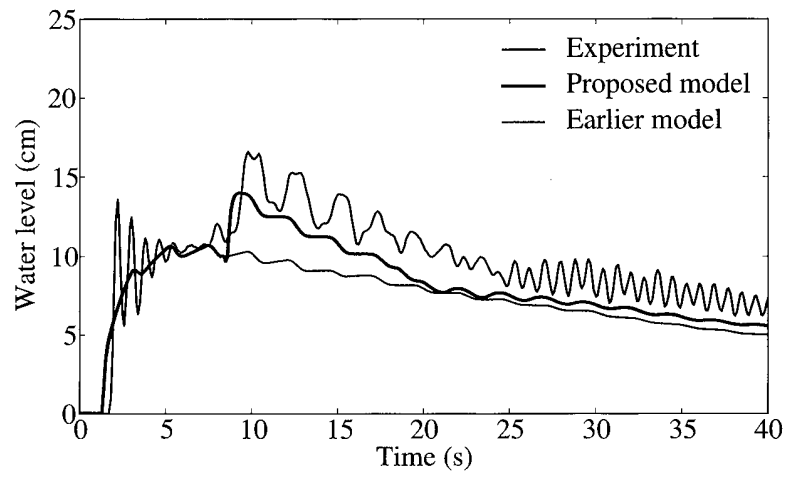

d) G4

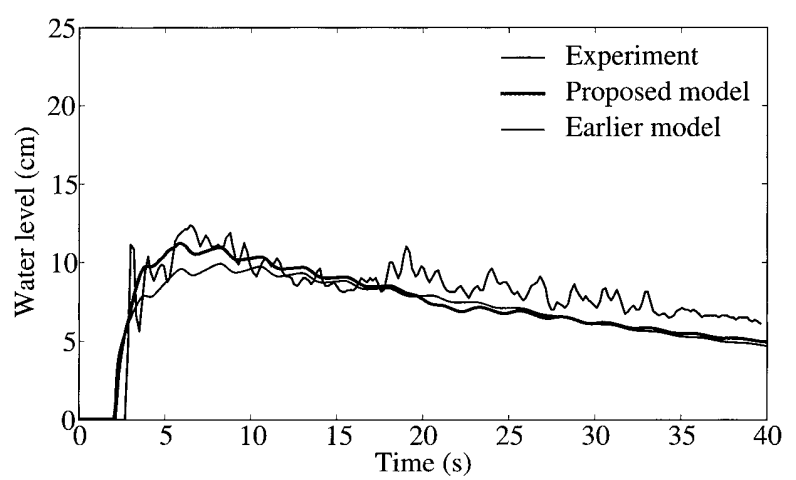

f) $\mathrm{G} 8$

Figure 6: Computed water levels by proposed and earlier models and experimental water level (Frazão et al., 1999) at the seven gauging points in the $45^{\circ}$ bend channel 
the hydraulic jump that occurred before the bends has also been compared between the computed and the experimental results. The position in the experimental results fluctuated because the wave caused by the hydraulic jump reflected on the wall of the bend. However, the computed results were within the range of the experimental ones. For validation in unsteady state, the water level of the dam break flows for the channel with a $90^{\circ}$ bend and that with a $45^{\circ}$ bend were compared between the computed results and the experimental data. The computed results agree with the experimental data well, although the computed celerity of the wave reflected on the bend is higher than the experimental one in the $45^{\circ}$ bend channel. Moreover, the proposed model has been compared with the earlier model neglecting the effects of bends. It is shown that the proposed model can reconstruct reflecting waves and backwater effects that the earlier model cannot. Consequently, the proposed model successfully reconstructs flows in bend channels.

\section{References}

[1] Biscarini, C., Francesco, S. D., and Manciola, P (2010): CFD modelling approach for dam break flow studies, Hydrol. Earth Syst. Sci., 14(4), pp.705-718.

[2] Ferrari, A., Dumbser, M., Toro, E.F., and Armanini, Aronne. (2009): A new 3D parallel SPH scheme for free surface flows, Computers $\mathcal{E}$ Fluids, 38, pp.1203-1217.

[3] Frazão, S.S., Sillen, X., and Zech, Y. (1999): Dam-break flow through sharp bends physical model and 2D Boltzmann model validation, Proc. CADAM meeting, Wallingford, United Kingdom, 2 and 3 March 1998, Commission Europenne, Bruxelles, pp.151-169.

[4] Frazão, S.S., and Zech, Y. (2002): Dam break in channels with $90^{\circ}$ bend, J. Hydr. Engrg., ASCE, 128(11), pp.956-968.

[5] Ishida, K., Yangyuoru, M., Unami, K., and Kawachi, T. (2011): Application of shallow water equations to analyze runoff processes in hilly farmlands, Paddy Water Environ., 9(4), pp.393-401.

[6] Kim, H., Lee, J.W., and Cho, Y.S. (2010): Numerical simulation of shallow-water flow using modified Cartesian cut-cell approach, J. Engrg. Mech., 136(3), pp.399-404.

[7] Liang, Q., Borthwick, A.G.L., and Stelling, G. (2004): Simulation of dam- and dyke-break hydrodynamics on dynamically adaptive quadtree grids, Int. J. Numer. Meth. Fluids, 46, pp.127-162.

[8] Sanders, B.F., Green, C.L., Chu, A.K., and Grant, S.B. (2001): Case study: Modeling tidal transport of urban runoff in channels using the finite-volume method, J. Hydr. Engrg., ASCE, 127(10), pp.795-804.

[9] Unami, K., Kawachi, T., Okumura, H., and Hiramatsu, K. (1996): Numerical model of pseudo steady flows in estuarine open channel networks using finite volume and finite element methods, Finite Volumes for Complex Applications, pp.563570 .

[10] Zhang, Y., Zeng, Z., and Chen, J. (2011): The improved space-time conservation element and solution element scheme for two-dimensional dam-break flow simulation, Int. J. Numer. Meth. Fluids. (in press).

[11] Zhou, J.G., Causon, D.M., Mingham, C.G., and Ingram, D.M. (2004): Numerical prediction of dam-break flows in general geometries with complex bed topology, J. Hydr. Engrg., ASCE, 130(4), pp.332-340.

Discussion open until December 31, 2012 